\title{
El mito de la sociología como «ciencia multiparadigmática» ${ }^{1}$
}

\section{The myth of sociology as a «multiparadigmatic» science}

\author{
José A. NoGUERA \\ Universidad Autónoma de Barcelona \\ jose.noguera@uab.cat
}

RESUMEN. En la sociología y la teoría social contemporánea se ha instalado profundamente la idea de que la sociología es inherentemente una ciencia o disciplina «multiparadigmática», y de que no es posible ni deseable caminar hacia una mayor integración en la disciplina. Este trabajo argumenta contra esa creencia y defiende la posibilidad y deseabilidad de establecer unas «reglas del juego» comunes como requisito lógico e institucional para perseguir fértil y críticamente la generación de conocimiento sociológico. Para ello, se efectúan algunas distinciones analíticas útiles: en primer lugar, entre pluralismo teórico genuino y pseudo-pluralismo; en segundo lugar, entre integración léxica, semántica, metodológica, epistémica y teórica; $y$, en tercer lugar, entre integración «simple»e integración «compleja» o «consiliencia». Adicionalmente, el artículo presenta algunas hipótesis para explicar la popularidad de este mito entre los sociólogos, entre las que destaca su uso como blindaje contra la crítica.

Palabras clave: paradigmas, integración teórica, epistemología, teoría sociológica, sociología analítica, pluralismo teórico.
ABSTRACT. Contemporary sociology and social theory are deeply penetrated by the idea that sociology is by its own nature a «multi-paradigm» science or discipline, and that it is not possible nor desirable to advance towards a higher degree of integration. This article argues against that belief, and defends the possibility and desirability of establishing a set of shared «rules of the game», as a logical and institutional condition in order to produce sociological knowledge in a fruitful and critical way. To that aim, some useful analytical distinctions are made: first, between genuine theoretical pluralism and pseudo-pluralism; second, between lexical, semantical, methodological, epistemic, and theoretical integration; and third, between «simple» and «complex» integration (or «consilience»). Additionally, the article presents some hypothesis to explain the popularity of this myth among sociologist; it is stressed that the myth may be used as a shield against criticism.

Key words: paradigms, theoretical integration, epistemology, sociological theory, analytical sociology, theoretical pluralism.

${ }^{1}$ El presente trabajo se ha desarrollado en el marco de un proyecto del Plan Nacional de $\mathrm{I}+\mathrm{D}+\mathrm{i}$ financiado por el Ministerio de Ciencia e Innovación (MICINN), con referencia CSO2009-09890. Una primera versión fue presentada como ponencia en el Grupo de Teoría Sociológica del V Congreso Catalán de Sociología (Barcelona, 17-18 de abril de 2009); el autor agradece a los asistentes a aquella sesión sus comentarios. 


\section{El mito: la sociología como «ciencia multiparadigmática»}

La mayoría de los estudiantes de sociología, normalmente durante su primer curso, han pasado por la experiencia de escuchar el siguiente discurso por parte de un desafiante profesor: «Creéis que la ciencia es una empresa enteramente racional e incontrovertible, pero no es así: existe algo, teorizado por un tal Kuhn, llamado "paradigma". El paradigma de turno es un conjunto de presuposiciones de diverso tipo que orienta todo el quehacer científico, es previo a éste, y no es racional en sí mismo; no está claro que un paradigma tenga que ser mejor que otro. En sociología, la cosa es aún más complicada, porque no existe un "paradigma dominante" ni puede existir; la sociología es por su propia naturaleza una "ciencia multiparadigmática"»".

En el peor (aunque no el más improbable) de los casos, lo anterior es sólo el preámbulo para la negación de la posibilidad misma de una sociología científica. A renglón seguido, se suele ilustrar a los mencionados estudiantes sobre los diversos «paradigmas» que existen en sociología, y sobre lo inevitable, e incluso deseable y enriquecedor, que es este «pluralismo» paradigmático. Si el profesor es astuto, irá además ofreciendo indicaciones directas e indirectas sobre cuál de esos muchos «paradigmas» es el «correcto» (porque sea el más «crítico», por ejemplo), y sobre qué adjetivaciones o denominaciones merecen quienes no lo compartan («positivistas», «economicistas», «psicologistas», «cientificistas», u otras por el estilo).

La idea de una «ciencia multiparadigmática» no fue, sin embargo, sugerida en modo alguno por Thomas Kuhn en el famoso libro que inauguró toda la floreciente industria académica sobre los «paradigmas», La estructura de las revoluciones científicas (1962). Según Kuhn afirmaba entonces, si una ciencia no cuenta con un paradigma dominante, entonces, simplemente, se trata de una disciplina pre-paradigmática, que no ha alcanzado la «madurez» como ciencia. Fue Margaret Masterman quien, en su comentario al libro de Kuhn (Masterman, 1974), acusó a éste de confundir un estado pre-paradigmático con uno multiparadigmático, definido este último como «ese estado de cosas en el que, lejos de no existir un paradigma, lo que hay, al contrario, son demasiados»; a ello añadía que «esta es la situación presente a grandes rasgos en las ciencias psicológicas, sociales y de la información» (ibid., 74).

La feliz ocurrencia de Masterman hizo fortuna inmediatamente en disciplinas como la sociología, y habrá pocos que discutan, hoy en día, que resulta un lugar común, en el inicio de cualquier texto o curso introductorio a la sociología, la teoría social o la epistemología de la ciencia social. Por poner algunos ejemplos que lo ilustren, baste citar a algunos teóricos sociales que son hoy considerados como pertenecientes al mainstream en estos campos. Para Anthony Giddens,

«La sociología no está dominada [...] por un único sistema conceptual.

Sin embargo, probablemente habría que considerar esto uno de sus puntos 
fuertes, no una de sus debilidades. No creo que esa diversidad haya producido un desorden completo, sino que, por el contrario, expresa el pluralismo que debe existir cuando se estudia algo tan complejo y controvertido como el comportamiento social humano y las instituciones» (Giddens, 1999, 16; cursivas mías).

En esta cita están presentes dos de las ideas-fuerza de lo que llamaré «el mito del carácter multiparadigmático de la sociología» (en adelante, simplemente «el mito»): 1) La complejidad social hace inevitable el pluralismo paradigmático en sociología. 2) Por tanto, o además, es bueno que esa pluralidad de paradigmas se perpetúe como situación habitual en la disciplina. En un conocido manual de teoría social, Giddens y Turner $(1987,12)$ llegan a decir que sólo en una sociedad totalitaria podría existir una ciencia social unificada.

Por su parte, el principal representante de la «teoría social crítica», Jürgen Habermas, argumenta en estos términos:

«la contienda de paradigmas tiene en las ciencias sociales un significado distinto que en la Física moderna. La originalidad de los grandes teóricos de la sociedad, como Marx, Weber, Durkheim y Mead, consiste (...) en que han introducido paradigmas que en cierto modo siguen compitiendo hoy en pie de igualdad. Estos teóricos siguen siendo contemporáneos nuestros, o en todo caso no se han vuelto históricos en el mismo sentido en que lo son Newton, Maxwell, Einstein y Planck, los cuales consiguieron progresos en el desarrollo teórico de un mismo paradigma básico. Por otro lado, los paradigmas guardan en las ciencias sociales una conexión interna con el contexto social del que surgen y en el que operan» (Habermas, 1981, I, 195).

Es obvio que para Habermas la coexistencia de diversos paradigmas en las ciencias sociales es un hecho conectado con la propia naturaleza de la sociedad como objeto de estudio, y además beneficioso para su comprensión, pues lo contrario, como ha defendido extensamente a lo largo de su obra (véase, por ejemplo, Habermas, 1982), supondría caer en una postura «positivista» de «reificación» de los hechos sociales; dado que resulta imposible «entender» esos hechos sin al mismo tiempo «valorarlos», se sigue que la irreductible diversidad de intereses y posiciones normativas conducirá por fuerza a distintos enfoques paradigmáticos, cada uno de los cuales captura alguna constelación de intereses y valores cognitivos y pragmáticos. La misma existencia de esos diversos paradigmas, afirma Habermas en un giro neohegeliano, nos da pistas insustituibles para entender la propia realidad social de la que surgen: en una subrepticia inversión de los términos, los «paradigmas» sociológicos acaban siendo para Habermas algo así como un «dato» para entender la sociedad, en vez de ser ésta la fuente de los datos con los que contrastar y construir teorías científico-sociales.

George Ritzer es uno de los sociólogos norteamericanos más leídos, y sus manuales de teoría sociológica aparecen virtualmente en todos los programas 
de esa asignatura en los estudios universitarios de sociología. Ritzer comulga con Habermas y Giddens al defender «la naturaleza multiparadigmática de la sociología (Ritzer, 2000, 614), a lo que añade que «la elección de un paradigma depende del tipo de cuestión que se aborde» (ibid., 621): habrá unos paradigmas más «adecuados» para estudiar un rango de fenómenos que otros. Una de sus obras tempranas se titula precisamente Sociology: A Multiple Paradigm Science (1975); en ella afirma que, dado que el llamado «pluralismo» es inherente a la naturaleza de la sociología, intentar lograr una síntesis es no sólo imposible, sino además indeseable, puesto que tal empresa mutilaría ese pluralismo (nótese la argumentación circular) y la supuesta «riqueza» que la sociología ha desarrollado en comparación con otras ciencias donde existe una «ortodoxia», que, al parecer, se entiende como «perjudicial» y «opresora» por definición (sin embargo, y sorprendentemente, ello no ha desanimado a Ritzer a la hora de defender su propio «paradigma metateórico integrado»: cfr. Ritzer, 2000, 614).

Otra de las «estrellas» del firmamento teórico-social contemporáneo, Jeffrey Alexander, se suma al consenso: afirma que en la ciencia (especialmente en la social) existe un «elemento apriorístico» que «no depende de las observaciones sino de las tradiciones» (Alexander, 1987, 15). La sociología «se desarrolla, a mi entender, dentro de tradiciones que se dan por sentadas y no están sometidas a una evaluación estrictamente empírica» (ibid., 15), lo cual, se infiere, es para él una buena noticia. Según reconoce, los «modos diversos, a menudo antitéticos, de conceptualizar los componentes básicos de las ciencias sociales son el núcleo del debate teórico contemporáneo» (ibid., 15). Esto es, el núcleo del debate teórico en sociología no tiene que ver con qué teoría es más explicativa o predictiva de un mismo rango de fenómenos, sino con un debate entre «tradiciones» no evaluables empíricamente. La elección entre una u otra dependerá entonces de los «valores» a los que cada cual se adscriba, y no son los hechos ni el mundo objetivo los que podrán refutar una tesis que cada respectiva «tradición» haya sancionado como «correcta». ${ }^{2}$

Se podría continuar así indefinidamente. Hace pocos años, un monográfico de la European Journal of Social Theory (vol. 10, n. ${ }^{\circ}$ 2, 2007), revista académica especializada reconocida en los círculos internacionales de la teoría social, recogía diversas opiniones de sociólogos ilustres sobre una eventual unificación de la sociología, las cuales, en su mayoría, se adherían de uno u otro modo a lo que he llamado «el mito». Como resume Alain Caillé en su presentación del volumen, mientras que algunos contribuyentes al debate sostienen que «esta insuperable y ecléctica diversidad de discursos teóricos y metodologías es algo bueno per se, dado que nos permite meditar sobre la diversidad ontológica y la multidimensionalidad de todas las relaciones socia-

2 Puede verse este argumento aún más abiertamente expuesto en otro trabajo reciente de Alexander (Alexander y Reed, 2009). 
les» (Caillé, 2007a, 181), otros cifran la imposibilidad de una unificación en sociología en «su inexpugnable dependencia contextual en la nación y la cultura» (ibid.). Y aun otros son lo bastante coherentes y sinceros como para no ocultar la consecuencia epistémica que ello tiene: «Concibo la sociología como una disciplina y no como una ciencia. Comparte este estatus con la filosofía y la historia. Las tres deben ser asignadas a una clase de disciplinas distinta de aquélla en la que se sitúan el estudio científico de la economía, la política, la religión, la demografía, y similares» (Baechler, 2007, 200). Como resignadamente observa Caillé, ante la consagración de la fragmentación y la heterogeneidad como señas de identidad, "one would have to conclude that the discipline exists only in the form of a fiction» (Caillé, 2007b, 277). ${ }^{3}$

En el resto de este texto, me propongo cuestionar este mito, y defender la tesis de que esta supuesta «peculiaridad» de la sociología respecto de otras disciplinas científicas no es ni necesaria o inevitable, ni mucho menos deseable. Argumentaré que el concepto mismo de «paradigma» está mal definido y resulta poco útil epistémicamente hablando, y que su aplicación a las ciencias sociales ha sido, como mínimo, discutible.

Para ello, permítaseme hacer una aclaración previa: como ya hemos visto, lo que llamo «el mito» puede tomar dos versiones, una positiva o fáctica, y otra normativa. Bajo la versión fáctica, el mito podría a su vez tomar dos formas, una de ellas sin interés: podría decir, en primer lugar que siempre es posible en una disciplina científica la existencia de diversos paradigmas. Considero que ésta es una tesis trivialmente verdadera: dada la vaguedad del concepto de paradigma, bastaría que un solo científico excéntrico elaborase un nuevo lenguaje conceptual para referirse a los fenómenos estudiados por su disciplina para hacer aparecer un «paradigma» diferente. Sin embargo, y en segundo lugar, existe una posible versión fáctica no trivial del mito: en este caso, lo que se sostendría es que es inevitable la existencia de diversos paradigmas en una disciplina, puesto que ello es algo inherente a la naturaleza de la misma y/o de su objeto de estudio. Esta tesis no es trivial, aunque es una forma de esencialismo ontológico y epistémico cuyas implicaciones me propongo mostrar.

La versión normativa del mito tampoco es trivial, pero es, creo, igualmente cuestionable: según ella, el pluralismo paradigmático en sociología es en sí mismo deseable, y debe ser cultivado y preservado como algo valioso, tal y como se prosiguen reverentemente las tradiciones religiosas y culturales. En un texto que hizo fortuna hace unas décadas en la sociología de las organizaciones, Burrell y Morgan (1979) capturaban y defendían explícitamente una actitud que ha hecho fortuna entre los sociólogos contemporáneos: el

3 Esta discusión sobre la fragmentación y la «crisis» de la sociología es recurrente. Un debate similarmente significativo puede verse en Sociological Forum, vol. 9, n. ${ }^{\circ} 2$ (1994) bajo el ilustrativo título «What's Wrong with Sociology?». 
«aislacionismo» paradigmático, la bondad de que cada paradigma avance solo, se haga internamente coherente, y permanezca indiferente a los demás, siendo sólo «verdadero para él mismo». «La auténtica necesidad», afirmaban, «es la clausura paradigmática. (...) Para evitar la castración y la asimilación (...), los paradigmas necesitan proveer una base para su autopreservación mediante el desarrollo de su propio enfoque» (Burrell y Morgan, 1979, 397398). Aunque a veces no se defienda tan clara y explícitamente, es difícil no advertir que buena parte de la mainstream sociology practica hoy día de facto esta recomendación.

\section{El concepto de paradigma y las ciencias sociales}

Resulta algo sorprendente que, a lo largo de los ríos de tinta a que ha dado lugar el libro de Kuhn, sea tan extremadamente difícil encontrar una definición lógicamente rigurosa del concepto de «paradigma». Cabe preguntarse, entonces: ¿está bien definido dicho concepto?; ¿resulta consistente su utilización?; ¿aporta algo epistémicamente útil?

Que el concepto de paradigma estaba muy vagamente definido en la obra de Kuhn ya lo notó Masterman (1974), quien contabilizó 21 significados distintos que éste podía tomar sólo en La estructura de las revoluciones cientificas (un texto, por lo demás, bastante corto). Pero una ojeada a la literatura sobre el tema y a la manera en que se utiliza el término en sociología no hará más que confirmar la impresión de que, en el fondo, no parece estar claro a qué nos referimos exactamente con esa expresión, a la que parece adscribirse un significado distinto según la conveniencia de quien la utilice. En el peor de los casos, se opera con una idea puramente intuitiva de «paradigma» que nunca se explicita como tal. Dependiendo de los textos y autores, «paradigma» se usa como sustituto y/o equivalente de cosas tales como escuelas teóricas, teorías, ideologías, principios metodológicos, tesis ontológicas, principios y valores epistémicos, o cualquier combinación o subconjunto de las anteriores.

En esta tesitura, cabría preguntarse qué aporta un concepto que prácticamente siempre puede traducirse por alguno de esos términos, mucho más claros y precisos, amén de ya conocidos y utilizados en la filosofía de la ciencia anterior a Kuhn. Se da la circunstancia de que el propio Kuhn (1962, 4-5) confirma esta impresión, pues cuando enumera los tipos de preguntas a las que un «paradigma» responde, la lista resultante se compone de cuestiones harto habituales y conocidas en la teoría de la ciencia:

- ¿Cuáles son las entidades fundamentales de que se compone el universo? (esto es, la cuestión ontológica).

- ¿Cómo interactúan con nuestros sentidos? (esto es, la epistemología y el estudio empírico de la cognición). 
- ¿Qué preguntas se pueden hacer legítimamente sobre ellas y qué técnicas se pueden emplear en buscar respuestas? (o sea, la cuestión metodológica).

- ¿Qué logros de la disciplina se convierten en modelos de la misma? (es decir, cuáles son las teorías centrales de la disciplina).

- ¿Qué problema es más significativo resolver? (a saber, como valorar epistémica y éticamente la relevancia cognitiva de cada línea de investigación).

No profundizaré aquí en la cuestión de cómo un «paradigma», o cualquier otra cosa, puede simplemente dictar la respuesta a todas esas preguntas de forma previa a (o independiente de) las teorías científicas y la evidencia empírica disponible en una disciplina, pues, por poner un solo ejemplo, parece sorprendente pretender que los supuestos ontológicos de la misma pueden adoptarse sin ningún tipo de control empírico, ni que sea implícito, indirecto, o de puro «sentido común»; en el fondo, pocos han notado que Kuhn y la jerga de los paradigmas van de la mano con el irrealismo de Milton Friedman (1953) y la economía neoclásica en cuanto a los supuestos de las teorías, que no estarían sujetos a prueba empírica, y podrían así ser irrealistas (o incluso surrealistas).

Tampoco me extenderé en cuestionar el supuesto, aparentemente implícito en el discurso de los paradigmas, de que por fuerza existen algo así como clusters cerrados y excluyentes que agrupan a determinadas respuestas asociadas entre sí (y sólo a ellas) para cada una de esas cinco preguntas. Los contraejemplos son tan numerosos, y los mecanismos que pudieran acaso explicar tal organización de las ideas, tan implausibles y difíciles de imaginar, que se hace costoso tomar en serio tal pretensión.

Lo que interesa notar, sin embargo, es que si descomponemos un paradigma en cuestiones de ontología, epistemología, metodología, teoría, y ética cognitiva, entonces la necesidad del concepto desaparece, y, lo que es más importante, empezamos a poder dudar de la tesis kuhniana de la inconmensurabilidad de los paradigmas, puesto que cada cuestión concreta se puede discutir y eventualmente decidir en su plano de relevancia respectivo, sin encontrarnos con la aparentemente infranqueable barrera del «paradigma» extraño e intraducible. Sin embargo, y desafortunadamente, lo habitual es partir de que esa barrera existe por defecto, y de que los paradigmas son algo así como «presupuestos metateóricos» inevitables (Alexander, 1987; Ritzer, 2000, 609), a la manera de una cosmovisión esencial o una especie de «agenda oculta», agazapada detrás de cualquier teoría o hipótesis científica. Al decir de Burrell y Morgan $(1979,36)$, los paradigmas están «definidos por supuestos meta-teóricos muy básicos en relación con la naturaleza de la ciencia y de la sociedad»; conceptos hermanos, según ellos, serían «problemáticas», «realidades alternativas», «marcos de referencia», «formas de vida», o «universos 
de discurso». Obsérvese el enorme salto entre esta lista y las cuestiones enumeradas por Kuhn. Obviamente, no es un salto sin consecuencias.

Como se ha sugerido, la sociología cumple sobradamente con todos los rasgos que Kuhn consideraba típicos de la «ciencia pre-paradigmática» (no de una supuesta ciencia «multi-paradigmática») ${ }^{4}$ : existen frecuentes debates sobre los métodos legítimos, sobre los problemas relevantes y sobre los estándares para darles respuesta, debates que sirven más para definir la identidad de las diferentes «escuelas» que para lograr un consenso (Kuhn, 1962, 47-48). Por este motivo, el propio Kuhn consideraba problemática la aplicación del concepto a las ciencias sociales. La sociología parece haber logrado desarrollar los «síntomas» institucionales o la «cosmética» de una «ciencia normal» (journals especializados, títulos y departamentos universitarios, congresos periódicos, etc.), pero sin haber interiorizado sus auténticos rasgos epistémicos. De hecho, en la imagen kuhniana de la ciencia normal, los paradigmas eran «objeto de subsiguiente articulación y especificación bajo nuevas y más rigurosas condiciones» (ibid., 23). En la sociología, salvo honrosas excepciones, más bien lo contrario es lo cierto: los seguidores de un paradigma se dedican no a intentar ponerlo a prueba, sino a hacer exégesis de los «modelos canónicos» del mismo, a seguirlo como una tradición sagrada, o a «aplicarlo» a objetos muy concretos pero sin variar sustancialmente los principios del mismo. De modo que, contra lo que el mito supone, pueden plantearse serias dudas sobre la coherencia interna del concepto mismo de paradigma, como también sobre la idoneidad de su aplicación a la situación efectiva de la sociología. Valga recordar que, curiosamente, ninguno de los clásicos de la disciplina se refirió a nada parecido a los «paradigmas» en sociología.

\section{Pluralismo genuino vs. pseudo-pluralismo}

En esta sección sostendré que las defensas de lo que he llamado el mito en base a la bondad de un supuesto «pluralismo» (o «antidogmatismo») son en gran parte retóricas, y en la práctica esconden la negación del auténtico pluralismo. Como han argumentado Goldthorpe (2000, 2007a) o Klima (1972), la competición científicamente fértil entre diferentes teorías presupone el acuerdo epistémico y/o metodológico sobre los requisitos o condiciones mínimas de la construcción de teorías científicas racionales, esto es, un acuerdo sobre las «reglas del juego» bajo las que la competición de ideas debe desarrollarse (y, eventualmente, decidirse).

4 Para la discusión, ya antigua, sobre si las ciencias sociales deberían o no considerarse pre-paradigmáticas puede verse Van Parijs (1982), Van Rossum (1975), Merton (1949: 86-91), o Lammers (1974). 
El auténtico pluralismo teórico, esto es, la existencia de diversas teorías que compiten por explicar los mismos hechos o tipos de fenómenos, es algo consustancial a toda ciencia: la competición entre distintas teorías, en términos empíricamente decidibles, es una condición de posibilidad del progreso científico. Por ello, una de las tareas fundamentales de los científicos es formular explicaciones alternativas de los fenómenos bajo estudio, que permitan explorar nuevas posibilidades y examinar críticamente la evidencia disponible. Este saludable y necesario pluralismo teórico, sin embargo, no tiene nada que ver con lo que usualmente se considera como tal en sociología, y que es, más bien, pseudo-pluralismo: consiste este último en la «coexistencia pacífica» entre diversas teorías o enfoques autorreferentes, inasequibles a la evidencia empírica y a la crítica desde otras posiciones teóricas, y cultivadas por comunidades cerradas de adeptos, cual si de sectas religiosas o clubs de fans se tratase. Goldthorpe, por ejemplo, observa que el auténtico pluralismo

«debe ir acompañado de una vigorosa competición entre ideas, que a menudo sea incluso mortal. Por el contrario, el pseudo-pluralismo se caracteriza por el hecho de que "la confrontación de puntos de vista" no se organiza como una competición, ya sea porque las escuelas rivales saben protegerse contra los ataques "ajenos", ya porque sus "aproximaciones" se formulan de tal forma que resulten inmunes en gran medida a todo tipo de crítica». [Para evitar esta situación debe existir un] «consenso metodológico básico que determine cuáles son las reglas fundamentales y los criterios de éxito de acuerdo con los cuales debe guiarse la competición entre ideas» (Goldthorpe, 2000, 7).

El auténtico pluralismo teórico, el que hace competir las teorías entre sí convirtiendo las disputas en algo decidible racionalmente, ha hecho avanzar en los últimos años campos de investigación tan importantes para la sociología como el estudio del capital social, de la acción colectiva, de las redes sociales, o de la movilidad y el estatus social, por citar sólo algunos (Aguiar, De Francisco y Noguera, 2009; Berger et al., 2005; Goldthorpe, 2007b; Noguera, 2006). En cambio, el pseudo-pluralismo teórico tiene su tragicómico reverso en la futilidad de los omnipresentes (y frecuentemente abstrusos) intentos de «superación de dicotomías teóricas», que a la postre acaban dejándolo todo igual, excepto por la creación de una corte de comentaristas, exégetas y críticos amigables. Los cultivadores de tales pseudo-discusiones se resisten a determinar qué cuenta como evidencia a favor o en contra de una teoría, y a entender las teorías no como compromisos vitales, identitarios o ideológicos (como cosmovisiones, al fin y al cabo), sino como modelos explicativos alternativos de un mismo fenómeno o rango de fenómenos, revisables y refutables a la luz de la evidencia empírica potencial. Una variante habitual de este tipo de discursos es el eclecticismo «epistemológicamente correcto» que simplemente yuxtapone diplomáticamente algunos eslóganes y términos rituales propios de cada «paradigma» para intentar contentar a todos y a ninguno en 
particular (algo habitual en «teóricos sociales» tan leídos como Giddens, Alexander, Bauman, Ritzer, Beck, Bourdieu o Habermas; cfr. Van den Berg, 1998; Barbera, 2004 y 2006, o Goldthorpe, 2000, 2007a).

En estas condiciones, cabe suponer que la eventual disolución de los «paradigmas» de la sociología, y la conversión de ésta en «ciencia normal», no supondría crear ortodoxia o dogma alguno. Bien al contrario: supondría la desaparición de los existentes y la consolidación de la sociología como un campo intelectual riguroso y como disciplina científico-social en pie de igualdad con otras. El auténtico dogmatismo (aunque a veces se vista de «crítico»o de ir «a contracorriente») consiste en pretender mantener los propios universos discursivos como reinos de taifas impertérritos a la crítica externa e impermeables a la evidencia empírica y el progreso del conocimiento científico. La supuesta «riqueza» intelectual que ese pseudo-pluralismo implica es puramente retórica: se traduce en pobreza informativa y esterilidad científica. Es la pluralidad paradigmática descontrolada la que impide la auténtica pluralidad de ideas, al «proteger» artificialmente las tradiciones intelectuales como si fuesen especies amenazadas o en extinción, blindándolas contra la crítica.

\section{Modos de integración en la ciencia social}

\subsection{Integración léxica, semántica, metodológica, epistémica y teórica}

Pero, ¿a qué nos referimos cuando hablamos de «integración»? Ésta es otra de las cuestiones infra-analizadas en el debate sobre los «paradigmas» en la ciencia social. Como ya se ha insinuado, existen diversos niveles en los que dicha integración puede tener lugar. La anarquía cognitiva «multiparadigmática» ha conseguido una dispersión y fragmentación sin precedentes en todos ellos. Cabría distinguir, al menos, entre los siguientes modos de integración en una disciplina:

1) Integración léxica: utilización de un mismo vocabulario para referirse al objeto u objetos de estudio.

2) Integración semántica o conceptual: utilización de los mismos conceptos para referirse a ese objeto.

3) Integración metodológica, o acuerdo en cuanto al tipo de preguntas de investigación a construir y los procedimientos con los que darles respuesta.

4) Integración epistémica, o acuerdo sobre las condiciones de posibilidad de la obtención de conocimiento válido y verdadero.

5) Integración teórica, o acuerdo sobre los modelos y teorías concretas que permiten explicar los fenómenos que son objeto de estudio de la disciplina. 
La integración léxica y la semántica suelen ir de la mano, empírica e históricamente, por motivos obvios, aunque no tiene por qué ser así lógicamente hablando; podría haber (y puntualmente hay) integración léxica pero discrepancia en cuanto a la definición o el significado de algunos términos; similarmente, podría haber (y puntualmente hay) integración conceptual, pero discrepancia o usos distintos en cuanto a los mejores términos para designar un determinado concepto.

Lo que interesa aquí, sin embargo, es que cuando la fragmentación «paradigmática» se reduce única o primordialmente a los aspectos léxicos y semánticos (algo que, en sociología, ocurre en un grado notable), se producen numerosos efectos perjudiciales para cualquier disciplina científica que se precie. Para empezar, proliferan supuestos «nuevos paradigmas» que a veces son sólo léxicos diferentes, un nuevo lenguaje o jerga con el que «renombrar» todos los fenómenos sociales y conceptos sociológicos ya conocidos (por ejemplo, la teoría de sistemas de Luhmann satisface bastante esta descripción; cfr. Luhmann, 1984). Ni que decir que este género de discurso va en detrimento del auténtico trabajo científico, que es el de explicación de los fenómenos; como afirma Hedström,

«Desde un punto de vista explicativo, las etiquetas son que designemos las entidades y mecanismos son siempre de menor importancia en la medida en que sus características definitorias y su lógica operativa estén claramente articuladas» (Hedström, 2005, 154).

Valga decir que esta operación de mera «redescripción» o «re-etiquetaje» del mundo no tiene nada que ver con el significado original del término «paradigma» en Kuhn: para éste, los paradigmas describen hechos distintos, mientras que lo que se tiene por paradigmas disímiles en sociología a menudo son meras redescripciones, en un vocabulario distinto, de los mismos hechos; son puras reformulaciones o nuevos vocabularios, que caen en el fetichismo lingüístico de suponer que cambiando las palabras o las etiquetas cambian los hechos o sus explicaciones. Por el contrario, el único efecto que tal operación tiene (y quizá el único pretendido) es retórico o estético; de ahí que, a menudo, adscribirse a un paradigma u otro acabe siendo una cuestión de gusto o de afinidad personal. A la inversa, en la visión de Kuhn, un paradigma nuevo resuelve realmente ciertos problemas que otro no puede solventar.

Si la fragmentación léxica es poco más que una frivolidad intelectual, la fragmentación conceptual, sin embargo, es algo más serio. Significa que no hay acuerdo sobre cuáles son los conceptos adecuados para «capturar» de forma relevante el objeto de estudio, en este caso, los fenómenos sociales. De hecho, muchos de los teóricos del mainstream sociológico antes citados sostienen que éste es el punto central de la discrepancia entre «paradigmas» (por ejemplo, Giddens, Habermas, Ritzer o Alexander). Sin embargo, es éste un pésimo planteamiento científico: la única manera de juzgar y decidir racio- 
nalmente sobre la adecuación, validez y utilidad de los conceptos científicos pasa por comprobar la fertilidad y potencia explicativa de las teorías en las que éstos se articulan e insertan. Aislar «la discusión de los conceptos» de la tarea, propiamente científica, de poner a prueba las teorías lógica y empíricamente, es plantearse la labor teórico-social como una escolástica especulativa y vacía; pero lamentablemente esto es lo que defienden, de forma explícita, los teóricos citados (véase Goldthorpe, 2000, o Van den Berg, 1998). ${ }^{5}$

El pluralismo metodológico y el téorico, bien entendidos, son aceptables y sobradamente practicados en muchas disciplinas científicas. Sobre el auténtico pluralismo teórico ya me he extendido en la sección anterior, así que baste añadir ahora la siguiente observación: la proliferación de (y competición entre) teorías distintas que intenten explicar los mismos hechos, amén de ser condición necesaria del progreso científico, tiene no obstante como ideal regulativo y objetivo último (aunque, como nos enseñó Popper, probablemente inalcanzable) la integración teórica. En mi opinión, muchos de los discursos que pasan por «paradigmas» en sociología son (o pretenden ser) teorías strictu sensu (e incluso diría que algo parecido ocurre en el planteamiento original de Kuhn). Pensemos, por ejemplo, lo frecuente que es oír hablar de «el paradigma de la elección racional» (por la teoría de la elección racional), o «el paradigma crítico» (por la teoría de Marx o de la Escuela de Frankfurt sobre el funcionamiento del modo de producción capitalista). No estoy seguro de qué información añade aquí la palabra «paradigma»; más bien creo que lo que se consigue (y quizá se busca) es crear la impresión de que por debajo de las teorías hay un «algo más» misterioso, una especie de «agenda oculta» que sólo el filósofo «crítico» y «que sospecha» puede vislumbrar. Esto se me antoja un supuesto esencialista, ya criticado convincentemente por Popper (1994) como «el mito del marco» y por Davidson (1984) como el mito del «esquema conceptual» (a los que me referiré más abajo).

En cuanto al pluralismo metodológico, también bien entendido, no tiene por qué suponer ninguna merma para la cientificidad de una disciplina. La sociología puede disfrutar de una sana pluralidad de estrategias metodológicas y técnicas para responder sus preguntas de investigación (Aguiar, De Francisco y Noguera, 2009). Pero, y esto es lo importante, ese pluralismo no se debe entender como la existencia de lógicas de inferencia «distintas» o «especiales» de la sociología (que es como tradicionalmente se ha defendido por parte de los sociólogos aficionados al mito que aquí se critica); las buenas estrategias metodológicas, aunque se conjuguen en plural, comparten una misma lógica de inferencia científica (King, Kehoane y Verba, 1994; Lago, 2008).

5 Cosa bien distinta es trabajar en la adecuada y precisa definición de los conceptos científico-sociales con vistas a la optimización de su papel lógico dentro de teorías sociológicas relevantes y explicativas, tarea no sólo legítima, sino muy necesaria (y especialmente abandonada en sociología). 
Por último, la integración epistémica parece un objetivo deseable, al menos hasta un cierto punto que permita la generación efectiva de conocimiento fiable en las disciplinas científicas, y ello aunque pueda seguir abierto el debate filosófico sobre multitud de cuestiones lógicas y gnoseológicas, pero siempre, como ocurría con el pluralismo teórico, con la vista puesta en el ideal de unas reglas del juego compartidas.

Integración léxica, semántica y epistémica (al menos en un determinado grado) son condiciones necesarias del debate intelectual y científico genuino. Son posibles y deseables en ciencias sociales. La integración metodológica y la teórica son posibles, pero probablemente no sean deseables como estado habitual, sino como ideal regulativo de la ciencia y del conocimiento en general. Pero, como hemos intentado mostrar, no es este «pluralismo» el que la actual jerga «multiparadigmática» defiende y encarna.

\subsection{Integración simple y compleja}

Conviene hacer una última distinción entre lo que llamaré integración «simple» y «compleja». Recordemos la cita de Giddens que comentamos en la sección 1: en ella se decía que la pluralidad paradigmática era una consecuencia necesaria de la complejidad del fenómeno a estudiar por parte de la sociología, la realidad social (y a esta tesis se sumaban, al menos, Habermas, Ritzer y Alexander). Éste es un prejuicio típico de los sociólogos del mainstream: la idea de que la gran complejidad de los fenómenos exige teorías y disciplinas mucho más complejas aún para explicarlos. Pero se trata de un malentendido, como Wilson (1998) ha mostrado con solidez: precisamente la complejidad de la realidad (de la natural no menos que de la social) es lo que la ciencia trata de «reducir» con sus teorías para entender mejor esa realidad. Sin reducción de complejidad, no hay ciencia ni hay auténtica comprensión de esa complejidad. ¿Qué hace pensar a Giddens que el comportamiento social es más complejo que la estructura subatómica de la materia, que los mecanismos de la evolución biológica, o que la neurobiología cerebral? Si la existencia de una gran «complejidad» es argumento para entender como beneficiosa la coexistencia de paradigmas, entonces deberíamos aceptar sin más la coexistencia en pie de igualdad de creacionistas y evolucionistas en biología, o de animistas y neurobiólogos en el estudio del funcionamiento cerebral.

Ahora bien, «reducción», como argumenta Wilson (1998, 101ss, 127ss), no siempre equivale a «eliminación». La reducción de un rango de fenómenos a otro rango de fenómenos de nivel «micro» es perfectamente compatible con la imposibilidad de predicción a partir de ese mismo nivel «micro» de los fenómenos del nivel «macro». La estructura y actividad de las células, por ejemplo, es perfectamente «reducible» hoy en día a la estructura y actividad de los elementos químicos que las componen y de los principios físico-atómi- 
cos que gobiernan tales elementos; sin embargo, no es posible reconstruir, a partir de una cierta cantidad y composición de elementos químicos, el tipo de células que resultarán, debido, entre otras cosas, a la variabilidad enorme en las disposiciones posibles del núcleo y los orgánulos de la célula, y en los intercambios químicos de ésta con su entorno. Con 10 átomos de carbono son posibles 75 tipos de moléculas, con 40 átomos, 62 billones de moléculas. En ciencias sociales no ocurre algo diferente: un determinado patrón de comportamiento colectivo (como una norma social) puede ser reducido a un conjunto de creencias, deseos y estados mentales individuales dispuestos de cierto modo, pero en muchas ocasiones es imposible predecir, a partir de dicho conjunto, qué patrón agregado de conducta colectiva resultará, debido a las múltiples disposiciones y configuraciones que dichos estados mentales pueden realizar (incluyendo los efectos de las actitudes estratégicas que anticipan las acciones ajenas).

Pues bien, propongo llamar integración «simple» a la que tiene lugar cuando una teoría o disciplina es eliminada en favor de otra, e integración «compleja» a la que tiene lugar cuando una teoría o disciplina es reducida a otra, pero sin que sea posible eliminarla (como en los ejemplos que acabamos de ver). Viene esto a cuento de dos cosas: a) el segundo tipo de integración constituye un progreso científico tan respetable como el primero (Wilson lo denomina «consiliencia»); b) lamentablemente, los defensores del «mito» confunden ambos tipos de integración, y creen que deben resistirse a la integración «compleja» por temor a que sea en realidad integración «simple». Esto es, nuevamente, una confusión que alimenta el mito de la bondad e inevitabilidad del estado multiparadigmático.

\section{5. ¿Hacia la unificación de la ciencia social?}

\subsection{La posibilidad de la unificación}

Causa cierta perplejidad observar cómo los mismos teóricos que sostienen lo inevitable y deseable de la situación multiparadigmática, a menudo se lanzan a proponer su propia forma de «integración entre paradigmas», que busca superar esa misma situación. Como se ha sugerido anteriormente, lo más habitual es que se trate de propuestas que adolecen de al menos una de estas dos limitaciones (Van den Berg, 1998; Barbera, 2004): a) la creencia de que es posible integrar sin más todos los «paradigmas» o «escuelas» sociológicas porque «cada una tiene parte de razón» y son primordialmente complementarias (mucho de este «eclecticismo políticamente correcto» hay en los respectivos intentos «integradores» de Ritzer, Giddens, Bourdieu, Alexander o Habermas); b) la creencia de que la tarea consiste simplemente en yuxtaponer o 
acumular teorías y supuestos con cierto maquillaje retórico, pero sin llevar a cabo una integración teórica formal. ${ }^{6}$

No es menos curioso que quienes defienden el mito lo suelen hacer, como se ha visto, sobre la base de la «naturaleza histórica y social» de las propias ciencias sociales, o de la relatividad espacio-temporal y hermenéutica de los significados subjetivos que «construyen» la realidad social. Pero, en cambio, por lo que al propio mito respecta, la postura de estos autores vira hacia un esencialismo a-histórico, merced al cual el carácter «multiparadigmático» de la sociología estaría inscrito en su propia naturaleza, en vez de ser la consecuencia de un proceso histórico contingente de institucionalización anómala (como han mostrado convincentemente Eisenstadt, 1978, o Stinchcombe, 1994). En este caso sí que estaríamos, por lo visto, ante una necesidad ontológica y epistémica (una de las pocas, todo sea dicho, que parecen admitir).

Esta supuesta necesidad ontológica que con tanta facilidad asumen los defensores del mito no tiene otra función que la de respaldar la versión positiva «fuerte» del mismo: la de que no es posible una integración teórica real en sociología, y por tanto la fragmentación de paradigmas es irrebasable. Ya se ha sugerido que existen contraejemplos suficientes en la práctica de la buena sociología científica como para refutar dicha tesis. Pero interesa ahora advertir que la misma es problemática no sólo desde el punto de vista empírico, sino también desde el conceptual. La razón ya la mostró Quine (1969): no pueden existir teorías científicas «no integrables» si se toman en el sentido de «alternativas equivalentes», pues, en tal caso (que es el relevante), o bien la disputa es decidible con criterios de predicción empírica y/o valores epistémicos como la simplicidad, la coherencia, etc., o bien las teorías son la misma en una formulación distinta (con lo cual estaríamos ante una simple diferencia léxica o semántica).

La versión del mito que ahora nos ocupa tiene su raíz última en las tesis así criticadas por Quine, críticas remachadas después por Davidson y Popper. Desgraciadamente, muchos sociólogos aún no son conscientes de las implicaciones de estas críticas. En «El mito del marco» (1994), Popper combate la extendida idea según la cual la verdad es relativa a nuestro trasfondo intelectual (a un «paradigma» o «cosmovisión»), del que se supone que determina lo que somos capaces de pensar; de ahí se seguiría la imposibilidad de comprensión mutua entre diferentes «marcos»; sería imposible la discusión fructífera si no se comparte al menos uno de ellos (Popper, 1994, 45-46). Pero a Popper no le cuesta mucho mostrar que dicho mito impone la falsa necesidad de aceptar o rechazar «el marco entero» en vez de poner a prueba y contrastar cada una de sus afirmaciones empíricas o teóricas en los contextos en que se planteen. Quizás más interesantemente aún, pero capturando similar argu-

6 Véase Gintis (2006) para los fundamentos teóricos de una ciencia social auténticamente integrada. 
mento, Davidson identifica lo que denomina «la idea de un esquema conceptual» del siguiente modo:

«Los esquemas conceptuales, se nos dice, son modos de organizar la experiencia; son sistemas de categorías que dan forma a los datos de los sentidos; puntos de vista desde los cuales los individuos, las culturas o las épocas contemplan el escenario en curso. Puede que no haya traducción de un esquema a otro (...). La realidad misma es relativa a un esquema: lo que cuenta como real en un sistema puede no serlo en otro» (Davidson, 1974, 183).

Esto es muy cercano, por no decir idéntico, a la noción kuhniana de paradigma. Sin embargo, como dice Davidson, con estas tesis suele pasar que «es difícil mejorar su inteligibilidad al mismo tiempo que se retiene su atractivo» (ibid.). Y ello por una razón: identificar algo como un «esquema conceptual» (y hacerlo desde otro «esquema conceptual», desde dónde si no) implica ya que la completa intraducibilidad entre ambos no existe. ¿Cómo determinar siquiera que lo que dice un paradigma es en alguna medida diferente de lo que dice otro, si no puedo «traducirlo» en los términos del segundo, o si no dispongo de un tercer código al que reducir las proposiciones de ambos? De modo que la supuesta relatividad a un esquema conceptual previo de la verdad de una afirmación acaba siendo la, mucho más familiar, relatividad a un lenguaje particular: «En vez de vivir en mundos distintos, los científicos de Kuhn podrían estar, como quienes necesitan el diccionario Webster, separados tan sólo por palabras» (Davidson, ibid.). Una vez más, estaríamos ante una mera cuestión léxica o semántica.

\subsection{La deseabilidad de la unificación}

Pero una fragmentación «paradigmática» como la que vive la sociología no sólo no es en absoluto inevitable, sino que es también indeseable. Goldthorpe (2000, 9), Stinchcombe (1994) y Folmer (2009) aducen importantes motivos que me permito sintetizar a continuación:

1) No poder apelar a un cuerpo establecido de procedimientos y conocimientos que «todo sociólogo debería poseer», y que se puede esperar que posea, conduce a numerosas ineficiencias, injusticias e inconsistencias. Por ejemplo: la dificultad de tomar decisiones eficientes sobre personal y plantillas académicas o científicas; los desacuerdos radicales a la hora de medir la productividad científica; la desigualdad en los raseros para medir los méritos en las evaluaciones académicas, en la selección de artículos y proyectos de investigación, o en las cualificaciones de los estudiantes; la imposibilidad de acordar lo que debería formar parte de un curso introductorio a la disciplina, etc. Por estos y otros motivos, las disciplinas que carecen de ese corpus serán las más vulnerables a recortes presupuestarios y re-estructuraciones burocráti- 
cas de diverso tipo. No estamos, por tanto, ante una cuestión que sólo tenga efectos lógicos y epistémicos, sino que también los tiene institucionales. Una disciplina que no comparte ni siquiera un mismo léxico o un mismo conjunto de definiciones, como nota Abbott (2001), da lugar a constantes malinterpretaciones, divisiones internas, y discusiones interminables sobre cuestiones no sustantivas, lo cual no es precisamente conveniente para lograr su reconocimiento social y político.

2) La dificultad de acordar criterios de excelencia para juzgar el trabajo sociológico tiene también consecuencias para la honestidad intelectual que debe presidir cualquier tarea académica. Dado que se tiene por imposible alcanzar un consenso intelectual genuino, los recursos materiales y simbólicos se acaban negociando y repartiendo, implícita o explícitamente, entre distintas «escuelas» o «sensibilidades», de acuerdo con criterios políticos y de forma epistémicamente hipócrita (véase Abbott, 2001, y Tarrow, 2008, para un análisis de casos concretos en este sentido). Esta situación, cuando hay financiación pública de las instituciones académicas, como suele ser el caso en muchos países, resulta doblemente deshonesta.

3) Por último, la fragmentación y el «todo vale» estimulan un «pickand-mix package approach to policy making» (Folmer, 2009, 264), según el cual los políticos y los gobernantes pueden abusar de la ciencia social escogiendo asesorarse sólo mediante aquellos grupos de investigación o aquellas aportaciones que confirmen sus preconcepciones y convengan a sus necesidades o intereses políticos. Esto es algo que no siempre ha sido adecuadamente tenido en cuenta por los sociólogos, pero que es decisivo para entender por qué la actual situación de fragmentación paradigmática es perniciosa. Desgraciadamente, incluso es habitual que se considere como una virtud la parcialidad política de una determinada escuela o enfoque, y que se seleccionen equipos de investigación que «comulguen» políticamente con quienes financian y encargan los estudios, muchas veces con el dinero de todos los contribuyentes.

En suma, ¿qué se gana en la sociología con el «pluralismo paradigmático»? De por sí, éste no aporta ni mayor informatividad a la disciplina, ni más capacidad explicativa o predictiva, ni mucho menos una mayor claridad y precisión en sus aportaciones. Quien gana es el escolasticismo y el academicismo, y quienes triunfan son los adeptos a la prosecución de tradiciones puramente discursivas o exegéticas, a la «traducción» entre pseudo-lenguajes y a la «pseudo-integración» teórica como deporte intelectual. Pero, desde un punto de vista científico, esta situación supone enormes pérdidas de tiempo, además del dramático despilfarro del talento y el esfuerzo de generaciones de jóvenes sociólogos que podrían estar produciendo auténtico conocimiento y auténtica teoría sociológica. 


\section{6. ¿A qué responde la popularidad del mito?}

Permítasenos aventurar, para acabar, algunas hipótesis que intenten dar cuenta de la popularidad de lo que he llamado «el mito» entre los sociólogos actuales.

a) Legitimación de la «soft social theory» como ocupación. Como afirma Goldthorpe, la idea de que la integración es imposible y/o indeseable

«congenia mucho con aquellos teóricos que creen que la principal tarea de la escritura teórica no es la de proveer una base para explicaciones más poderosas, sino más bien la de comentar, analizar y criticar "discursivamente" la variedad de tradiciones y escuelas que la sociología comprende. Pues, de ese modo, se abre un panorama de extensión infinita para que se escriban libros a partir de otros libros, con escasa necesidad de investigación empírica» (Goldthorpe, 2000, 7).

Así, la sociología quedaría cómodamente reducida a una labor de ensayismo o crítica e interpretación de textos, que sin duda exige un coste mucho menor en adquisición de habilidades técnicas, y provee de beneficios mucho mayores y más rápidos que la paciente y siempre incierta investigación científica.

b) Corporatismo profesional «pragmático». Aceptar y contemporizar con la anarquía intelectual de facto y llamarla «pluralismo» puede ser, en muchos casos, «la única manera de preservar un mínimo de cohesión socio-política» en la profesión (Golthorpe, 2000, 7), de modo que se obtengan recursos y cierto reconocimiento social. Existiría un equilibrio estratégico implícito: "yo no me meto con tu paradigma si tú no te metes con el mío», de forma que obviemos toda discusión al respecto cuando de presentarnos corporativamente para obtener recursos y reconocimiento se trata. Como se ha sugerido, se trataría en este caso de un acuerdo de intereses negociado políticamente, no alcanzado argumentativamente sobre bases epistémicas fiables. Nada bueno cabe deparar a una ciencia que se institucionaliza de ese modo y que se estanca complacientemente en tal situación. Ésta puede ser un equilibrio político-estratégico, pero en términos epistémicos es claramente subóptima.

c) Blindaje contra la crítica y la refutación. Refugiándose en un "paradigma» es posible resguardarse de la crítica de los demás. Nótese el carácter circular del tipo de «sociología» que se suele hacer desde estos «paradigmas»: se suele encontrar aquello que las «presuposiciones teóricas» ya han decidido que se va a encontrar. La teoría crítica y los foucaultianos por fuerza encontrarán opresión y disciplina por doquier (porque es su «supuesto teórico»), los luhmanianos encontrarán autopoiesis, los interaccionistas encontrarán negociación de definiciones de la situación, los seguidores de Bourdieu efectos de campo y de habitus, los giddensianos procesos de estructuración y reflexive monitoring, los posmodernos fragmentación y disolución de lo social, etc. Los resultados de cualquier investigación empírica desde uno de ta- 
les paradigmas siempre podrán ser interpretados de forma que encajen con los postulados y presuposiciones del mismo, y por tanto refuercen su «validez».

Pero los paradigmas no sólo sirven para construir el propio blindaje, sino para disponer de proyectiles dialécticos listos para lanzar contra cualquier afirmación hecha «desde fuera»; el aprendizaje de los mismos constituye parte esencial en la iniciación de los estudiantes en cada paradigma. De esa forma, cuando un científico social diga (por poner un ejemplo) que «el nivel de confianza social generalizada depende de la densidad de las redes sociales en las que los individuos están insertos», la crítica automática a esa afirmación empírica se basará en «buscar sus presuposiciones metateóricas» (por ejemplo, por partir de una concepción individualista o reticular de la sociedad, o de una concepción objetivista de la ciencia social, o de una metodología «psicologista»). Nótese que ni siquiera se habrá entrado a discutir la tesis misma en sus aspectos sustantivos ni empíricos: eso no será necesario. Basta «identificarse» con un paradigma para tener lista una crítica automática de los demás discursos, pues no se trata más que de denunciar unos «presupuestos metateóricos» desde otros, con lo cual la crítica se convierte en una trivialidad.

Los paradigmas se convierten así en el mejor blindaje imaginable contra la crítica, y en una fuente inagotable de diálogos de sordos. Si se acepta que los paradigmas son «inconmensurables», entonces va de suyo la idea de que no puede haber crítica con sentido entre unos y otros (lo cual hace más curioso que quienes suscriben esa tesis se embarquen a menudo en tales «críticas»). La consecuencia práctica de esto es que el mito funciona como un incentivo para el atrincheramiento detrás de un paradigma, sea el que sea. La regla para los avezados es: «escoge un paradigma, y cuando no sepas o no puedas responder a una crítica, escúdate en él». De este modo, se extiende la visión de la sociología como "armario guardarropa» donde uno puede escoger el traje que le «quede» mejor, donde la elección de paradigma o de teoría es una cuestión de gusto o de intuición subjetiva, o, para los más eclécticos, de conveniencia percibida de cada estudio o investigación concreta.

Sin embargo, una regla de sentido común de la justificación racional de creencias o tesis cuando se trata de convencer de las mismas a quienes no las comparten es la de proceder de forma «metafísicamente ligera» (a la manera de Rawls en su teoría de la justicia o del consenso entrecruzado): evitar premisas o supuestos «fuertes», inherentemente controvertidos, de tipo metafísico, ideológico o especulativo; tender a partir de hechos claros y comúnmente aceptados o de premisas compartidas o de sentido común, e intentar moverse desde allí a las conclusiones que se desea justificar. Ahora bien, el discurso y la actitud de los paradigmas preconiza habitualmente lo contrario: partir precisamente de aquello que el oponente no pueda aceptar, de forma que no haya nada más que probar, justificar o discutir; se trata de esgrimir el «paradigma» desde el principio como bandera y como protección frente a cualquier hecho 
palpable o premisa de puro sentido común que el oponente aporte a la discusión y que no convenga considerar. Así se conseguirá el auténtico objetivo: mantener la pureza inmaculada del paradigma y de los intocables «supuestos metateóricos». Si además ello se adereza con amables declaraciones sobre el «diálogo» y la «integración» entre paradigmas en todo lo que tengan de «complementario», el éxito habrá sido completo.

Dicho de otro modo, el discurso de los paradigmas parece claramente diseñado para evitar que se puedan producir acuerdos racionales, porque allí donde éstos son posibles, se puede tener que acabar renunciando o modificando algunas de las premisas más queridas, y eso resulta inaceptable para quien se ha instalado en un paradigma como una cuestión de identidad personal, de fidelidad a una comunidad tribal, o de ideología política o académica.

\section{A modo de conclusión}

Los defensores del mito pueden responder (y frecuentemente lo hacen) con la estrategia del tu quoque: todo lo que se dice contra el discurso de los paradigmas estaría basado en otro paradigma, con lo que en el fondo lo único que se intenta es imponer el propio, exterminando de forma imperialista la necesaria y deseable pluralidad y libertad de pensamiento, y otros perjuicios por el estilo. Es éste el tipo de razonamiento de los creyentes religiosos que no aceptan que existan ateos (porque éstos también «creen» en el fondo en su propia religión), o de los nacionalistas que no aceptan que existan «ciudadanos del mundo» o personas agnósticas respecto de la pertenencia nacional (pues ellos también serían «nacionalistas» de alguna nación aunque no lo sepan o no lo quieran reconocer). Salta a la vista el juego de palabras: cuando se han agotado las estrategias de escape, lo único que resta es cambiar la definición de «paradigma» (o de «religión», o de «nación») sobre la marcha para que pueda incluir creencias que bajo la definición original no habría incluido, es más, que precisamente se oponen a la existencia misma de lo que el término pretendía designar. Dicha estrategia tiene también un aspecto ad hominem típico de muchos otros dogmas: quien cuestione el Paradigma lo que hace es probar con ello que tiene un serio problema de autopercepción por el cual no es capaz de «ver» su propio paradigma.

La conclusión es clara: ignoremos, disolvamos, el discurso de los «paradigmas». Como afirmaba Popper (1994), dado que los «marcos» o «paradigmas» se conciben habitualmente como cosmovisiones, formas de vida, o maneras de ver el mundo que constituyen un vínculo social o cuasi-religioso entre sus devotos, están en las antípodas del espíritu y del ethos del auténtico científico (incluido el científico social): éste tiene interés por las teorías decidibles empíricamente, no por las doctrinas cosmovisionales. A la manera de la interpretación radical davidsoniana, la propuesta de disolución de los para- 
digmas en sociología no es la de eliminar los desacuerdos, sino la de hacerlos inteligibles y significativos (Davidson, 1974, 196). Incrementamos el sentido mismo de las diferencias entre nuestras creencias cuando incrementamos su traducibilidad, y no al contrario. La idea de que las diferencias yacen en algún lugar oculto, «al fondo» o "por detrás» de esas creencias (en un «paradigma»), no añade nada esencial desde un punto de vista lógico; no puede haber una base inteligible a partir de la cual decir que nuestros «esquemas conceptuales» o «paradigmas» son diferentes, más allá de la que nos permite decir que nuestras afirmaciones, creencias o teorías sobre el mundo, sin más, son diferentes. Ya se ha notado que la artificiosa fragmentación de la Gesellschaft científico-social en multitud de pequeñas comunidades cerradas y autorreferenciales tiene importantes y variados costes que la sociología no debería asumir. Pero quizá el mayor de todos ellos sea la magnificación de las diferencias intelectuales irrelevantes y la ocultación de las realmente importantes.

\section{REFERENCIAS}

Aввотт, Andrew (2001). Chaos of disciplines. Chicago, University of Chicago Press. Aguiar, Fernando; De Francisco, Andrés y Noguera, José A. (2009). «Por un giro analítico en sociología», Revista Internacional de Sociología, vol. 67, n. ${ }^{\circ} 2$, pp. 435-456.

AleXANDER, Jeffrey C. (1987). Las teorías sociológicas desde la segunda guerra mundial. Barcelona, Gedisa, 1995.

AleXANDER, Jeffrey C. y ReED, Isaac (2009). «Social Science as Reading and Performance: A Cultural-Sociological Understanding of Epistemology», European Journal of Social Theory, vol. 12, n. ${ }^{\circ}$ 1, pp. 21-41.

BAECHLER, Jean (2007). «But What Is Sociology?», European Journal of Social Theory, vol. 10, n. ${ }^{\circ}$ 2, pp. 200-205.

BARBERA, Filippo (2004). Meccanismi sociali. Elementi di sociologia analitica. Bologna, Il Mulino.

- (2006). «A Star is Born? The Authors, Principles and Objectives of Analytical Sociology», Papers. Revista de Sociologia, n. ${ }^{\circ} 80$.

Berger, Joseph; Willer, David y Zelditch, Morris (2005). «Theory Programs and Theoretical Problems», Sociological Theory, vol. 23, n. ${ }^{\circ}$ 1, pp. 127-155.

BURRELl, Gibson \& MorGAN, G. (1979). Sociological paradigms and organisational analysis. Croft Road, Gower.

CAILlÉ, Alain (2007a). «Introduction to Symposium», European Journal of Social Theory, vol. 10, n. ${ }^{\circ}$ 2, pp. 179-183.

- (2007b). «Sociology as Anti-Utilitarianism», European Journal of Social Theory, vol. 10, n. ${ }^{\circ} 2$, pp. 277-286.

DAVIDSON, Donald (1984). «On the Very Idea of a Conceptual Scheme», en Inquiries into Truth and Interpretation. Oxford, Clarendon Press.

EISENSTADT, S. N. (1978). «La tradición sociológica», Revista Española de Investigaciones Sociológicas, $\mathrm{n}^{\circ}{ }^{\mathrm{1}}$, pp. 7-43. 
Elster, Jon (2007). Explaining Social Behavior. New York, Cambridge University Press.

Folmer, Henk (2009). «Why Sociology is Better Conditioned to Explain Economic Behaviour than Economics», Kyklos, vol. 62, n. ${ }^{\circ}$ 2, pp. 258-274.

Friedman, Milton (1953). «La metodología de la economía positiva», en Ensayos sobre economía positiva. Madrid, Gredos, 1967.

GidDEns, Anthony (1999). En defensa de la sociología. Madrid, Alianza, 2000.

Giddens, Anthony y Turner, Jonathan (eds.) (1990). La teoría social, hoy. Madrid, Alianza.

GINTIS, Herbert (2006). «Towards a Unity of the Human Behavioral Sciences», $P a$ pers. Revista de Sociología, n. ${ }^{\circ} 80$.

Goldthorpe, John H. (2000). On Sociology. Numbers, Narratives and the Integration of Research and Theory. Oxford, Oxford University Press.

- (2007a). On Sociology (Second Edition). Vol. 1: Critique and Program. Stanford, Stanford University Press.

- (2007b). «Progress in Sociology: The Case of Social Mobility Research», en Stefan Svallfors (ed.), Analyzing Inequality: Life Chances and Social Mobility in Comparative Perspective. Stanford, Stanford University Press, pp. 56-82.

HABERMAS, Jürgen (1981). Teoría de la acción comunicativa (2 vols.). Madrid, Taurus, 1987.

- (1982). La lógica de las ciencias sociales. Madrid, Tecnos, 1996.

Hedström, Peter (2005). Dissecting the Social. On the Principles of Analytical Sociology. Cambridge, Cambridge University Press.

Klima, Rolf (1972). «Theoretical Pluralism, Methodological Dissension, and the Role of the Sociologist», Social Science Information, vol. 11, pp. 69-108.

King, Gary; KeOHAne, Robert O. y Verba, Sidney (1994). Designing social inquiry: scientific inference in qualitative research. Princeton (N.J.), Princeton University Press.

KuHN, Thomas (1962). La estructura de las revoluciones científicas. México, Fondo de Cultura Económica, 1986.

LAGO, Ignacio (2008). La lógica de la explicación en las ciencias sociales. Madrid, Alianza.

LAMMERS, C. J. (1974). «Mono and poly-paradigmatic developments in natural and social sciences», en Richard WHITLEY (ed.), Social processes of scientific development. Routledge \& Kegan Paul, London, pp. 123-147.

LuHmann, Niklas (1984). Sistemas sociales. Lineamientos para una teoría general. Barcelona, Anthropos, 1998.

MAsterman, Margaret (1974). «The Nature of a Paradigm», en I. LAKATOS (ed.), Criticism and the Growth of Knowledge. Cambridge, Cambridge University Press.

Merton, Robert K. (1949). Teoría y estructura sociales. México, Fondo de Cultura Económica, 1968.

NoguerA, José A. (2006). «Why We Need An Analytical Sociological Theory», Papers. Revista de Sociología, n. ${ }^{\circ} 80$.

Quine, W. V. (1969). Ontological Relativity and Other Essays. Nueva York, Columbia University Press.

PopPeR, Karl R. (1994). «El mito del marco», en El mito del marco y otros ensayos. Barcelona, Paidós. 
Ritzer, George (1975). Sociology: A Multiple Paradigm Science. Boston, Allyn \& Bacon.

- (2000). Teoría sociológica moderna. Madrid, McGraw-Hill, 2002.

STINCHCOMBE, Arthur L. (1994). «Disintegrated Disciplines and the Future of Sociology», Sociological Forum, vol. 9, n. ${ }^{\circ}$ 2, pp. 279-291.

TARROW, Sidney (2008). «Polarization and convergence in academic controversies», Theory \& Society, vol. 37, pp. 513-536.

VAn DEN Berg, Axel (1998). «Is Sociological Theory Too Grand for Social Mechanisms?», in Hedström, Peter \& Swedberg, Richard (eds.): Social Mechanisms. An Analytical Approach to Social Theory. Cambridge, Cambridge University Press.

VAn PARIJs, Philippe (1982). Evolutionary Explanations in the Social Sciences, Totowa, New Jersey, Rowman and Littlefield.

VAN Rossum, Wouter (1975). «The problem of cognitive institutionalization in the social sciences: The case of Dutch sociology», Social Science Information, vol. 14 (29): 155-172.

Wilson, Edward O. (1998). Consilience. The Unity of Knowledge. New York, Vintage Books. 